\title{
Can the Mott Insulator TiOCl be Metallized by Doping? A First-Principles Study.
}

\author{
Yu-Zhong Zhang, ${ }^{1}$ Kateryna Foyevtsova, ${ }^{1}$ Harald O. Jeschke, ${ }^{1}$ Martin U. Schmidt, ${ }^{2}$ and Roser Valentí ${ }^{1}$ \\ ${ }^{1}$ Institut für Theoretische Physik, Goethe-Universität Frankfurt, \\ Max-von-Laue-Straße 1, 60438 Frankfurt am Main, Germany \\ ${ }^{2}$ Institut für Anorganische und Analytische Chemie, Goethe-Universität Frankfurt, \\ Max-von-Laue-Straße 7, 60438 Frankfurt am Main, Germany
}

(Dated: November 3, 2018)

\begin{abstract}
We investigate the effect of $\mathrm{Na}$ intercalation in the layered Mott insulator $\mathrm{TiOCl}$ within the framework of density functional theory. We show that the system remains always insulating for all studied $\mathrm{Na}$ concentrations, and the evolution of the spectral weight upon $\mathrm{Na}$ doping is consistent with recent photoemission experiments. We predict the Na-doped superlattice structures, and show that substitutions of $\mathrm{O}$ by $\mathrm{F}, \mathrm{Cl}$ by $\mathrm{S}$, or $\mathrm{Ti}$ by V (or Sc), respectively, fail to metallize the system. We propose a description in terms of a multiorbital ionic Hubbard model in a quasi-two-dimensional lattice and discuss the nature of the insulating state under doping. Finally, a likely route for metallizing TiOCl by doping is proposed.
\end{abstract}

PACS numbers: 71.15.Mb,71.15.Pd,71.27.+a,71.30.+h,71.70.Ch

A successful route to drive a layered compound into a (usually unconventional) superconducting state is by doping the system with additional electrons or holes [17]. In contrast to the cuprates [1, 2], Fe-based systems [3 5], and cobaltates 7] where 3d ions with occupations larger than 5 electrons are responsible for superconductivity, in the layered nitride superconductors [6], Zr and $\mathrm{Hf}$ are in $4 \mathrm{~d}^{2}$ and $5 \mathrm{~d}^{2}$ configurations. In this work we investigate whether unconventional superconductivity can be realized in early $3 \mathrm{~d}$ layered compounds upon doping. A good candidate is $\mathrm{TiOCl}$ which is, similar to the cuprates, a layered Mott insulator at room temperature [8] but with $\mathrm{Ti}^{3+}$ in its $3 \mathrm{~d}^{1}$ configuration; Ti is in the same column of the periodic table as $\mathrm{Zr}$ and $\mathrm{Hf}$. According to these features, a fundamental question is whether $\mathrm{TiOCl}$ can be a metal or even superconductor by doping.

In fact, the undoped TiOCl, formed by stacked bilayers of $\mathrm{TiO}$ along the $c$ axis separated by $\mathrm{Cl}$ ions, has already attracted considerable interest [9 13] due to the appearance of a zero-field structural incommensurate state. Furthermore, great effort has been put into metallizing the undoped TiOCl by applying hydrostatic pressure [14, 15].

Very recently, samples of $\mathrm{TiOCl}$ with $\mathrm{Na}$ intercalated into the interstices of the $\mathrm{TiO}$ bilayers were prepared in order to drive the system into a metallic or even superconducting state upon electron doping [16]. However, photoemission experiments (PES) show that a pronounced gap remains at the Fermi level for all concentrations of $\mathrm{Na}$, indicating that the insulating state always survives electron doping [16]. In view of these results, it is essential to understand the microscopic origin of this puzzling insulating state upon doping and, thereafter, find a more promising recipe for metallizing TiOCl. Moreover, considerable interest in insulator-insulator transitions [17] motivates us to investigate the nature of the

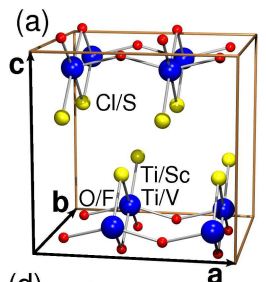

(d)
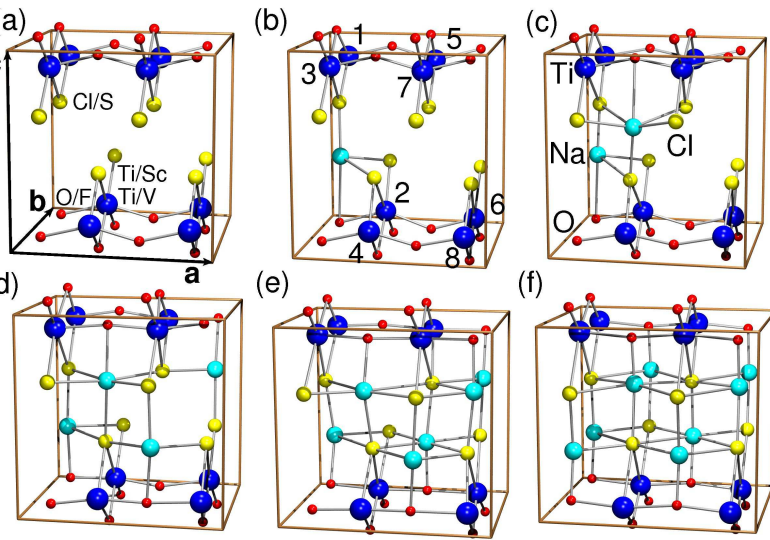

(e)

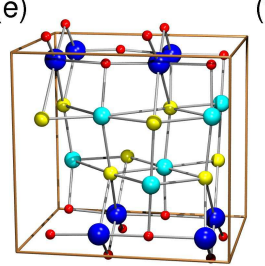

(f)

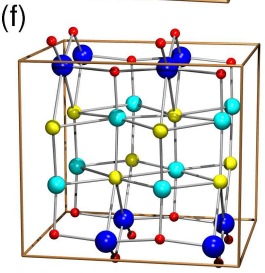

FIG. 1: (Color online) Superlattices at 6 different concentrations of Na doping. (a) undoped case. The atoms where substitutions of $\mathrm{Cl}$ by $\mathrm{S}, \mathrm{O}$ by $\mathrm{F}$ and $\mathrm{Ti}$ by $\mathrm{V}(\mathrm{Sc})$ are performed are also specified. (b) $12.5 \% \mathrm{Na}$ doping: $\mathrm{Na}_{1 / 8} \mathrm{TiOCl}$. The $8 \mathrm{Ti}$ ions in the cell are labelled by numbers 1 to 8 . (c) $25 \%$ doping: $\mathrm{Na}_{1 / 4} \mathrm{TiOCl}$, (d) $50 \%$ doping: $\mathrm{Na}_{1 / 2} \mathrm{TiOCl}$, (e) 75 \% doping: $\mathrm{Na}_{3 / 4} \mathrm{TiOCl}$, and (f) $100 \%$ doping: NaTiOCl.

doped insulating state.

In this Letter, we performed ab initio molecular dynamics studies with the Car-Parrinello [18] projectoraugmented wave (CP-PAW) method [19] to study the effect of $\mathrm{Na}$ doping on $\mathrm{TiOCl}$ within the ParrinelloRahman scheme [20]. The Perdew-Burke-Ernzerhof generalized gradient approximation $(\mathrm{GGA})+U$ to the density functional theory (DFT) is employed in order to have the correct insulating behavior and lattice structure in the undoped case [13, 15, 21 23].

Our findings can be summarized as follows: For all $\mathrm{Na}$ concentrations studied, the unusual insulating state persists and the change of spectral weight at elevated Na concentration agrees well with the PES results [16]. 


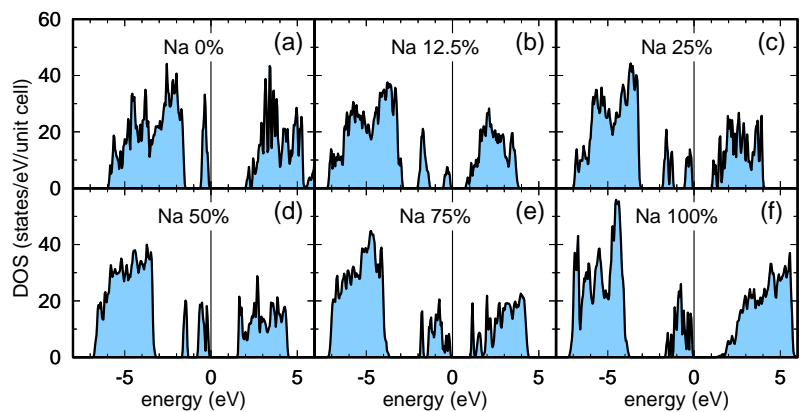

FIG. 2: (Color online) Total density of states (DOS) at the 6 different concentrations of $\mathrm{Na}$ doping shown in Fig. 1, (a) undoped case: $\mathrm{TiOCl}$, (b) $\mathrm{Na}_{1 / 8} \mathrm{TiOCl}$, (c) $\mathrm{Na}_{1 / 4} \mathrm{TiOCl}$, (d) $\mathrm{Na}_{1 / 2} \mathrm{TiOCl}$, (e) $\mathrm{Na}_{3 / 4} \mathrm{TiOCl}$, and (f) NaTiOCl .

Analysis of this insulating state shows that the apropriate microscopic model is no longer a dominantly quasi-one-dimensional single-band model valid in the undoped case [8, 11 13] but a two-dimensional multi-orbital model. The effect of Na doping is twofold: (i) it strongly modifies the crystal field splitting of Ti d states by the $\mathrm{Na}^{+}$ion and (ii) it induces a coexistence of $\mathrm{Ti}^{3+}$ and $\mathrm{Ti}^{2+}$ ions which is further stabilized by long-range Coulomb interaction as usually observed in mixed-valence compounds 24]. Together with the on-site Coulomb repulsion as well as the Hund's rule coupling, these effects play a crucial role in forming the doped symmetry-breaking insulating phase. We further predict the Na-doped superlattice structures, and we also find that doping TiOCl by substituting $\mathrm{O}$ by $\mathrm{F}, \mathrm{Cl}$ by $\mathrm{S}$, or Ti by $\mathrm{V}$ (or $\mathrm{Sc}$ ) does not result in a metal. While the insulating state of the $\mathrm{F} / \mathrm{S}$ substitution case can be ascribed to the same microscopic origin as in the Na-doped case, the long-range Coulomb interactions play a minor role in the $\mathrm{V} / \mathrm{Sc}$ substitution.

For our calculations we used supercells obtained by doubling the primitive unit cell (space group Pmmn, two formula units per unit cell) in both $a$ and $b$ directions. The molecular formula under doping is defined as $\mathrm{Na}_{x} \mathrm{Ti}_{1-y} \mathrm{~V}_{y}\left(\mathrm{Sc}_{y}\right) \mathrm{O}_{1-z} \mathrm{~F}_{z} \mathrm{Cl}_{1-w} \mathrm{~S}_{w}$, where $x, y, z$, and $w$ are the doping concentrations (Fig. 1(a)). We performed carefully converged relaxations of lattice parameters and atomic positions for each doping case with high energy cutoffs of 45 Ry and 180 Ry for the wave functions and charge density expansion, respectively.

We first study the case of $\mathrm{Na}_{x} \mathrm{TiOCl}$ for six different values $x=0,1 / 8,1 / 4,1 / 2,3 / 4,1$. Since no experimental data for the Na positions are available, numerous possibilities have to be tested by ab initio molecular dynamics. However, according to Pauling's rule that the coordination number is determined by the radius ratio [25], the most probable $\mathrm{Na}$ positions are in the cages of $5 \mathrm{Cl}$ and $1 \mathrm{O}$ as shown in Figs. 1(b)-(f). In fact, by performing $a b$ initio molecular dynamics starting from several different initial $\mathrm{Na}$ positions, we find that the $\mathrm{Na}$ atoms always fall into this cage to reach the coordination number of 6 .

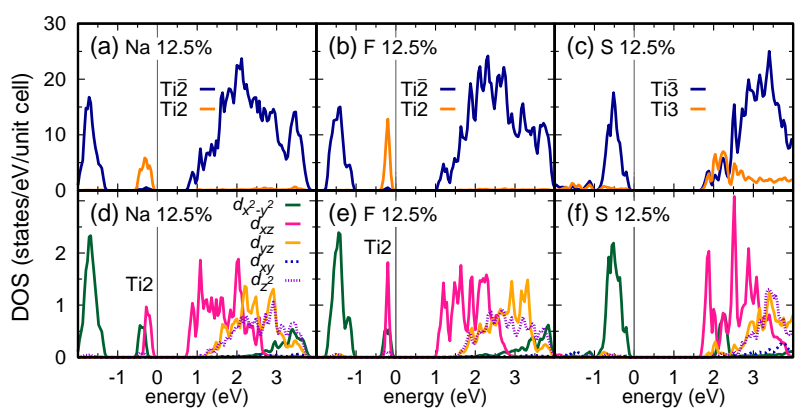

FIG. 3: (Color online) Partial DOS for (a), (d) $12.5 \%$ Na doping: $\mathrm{Na}_{1 / 8} \mathrm{TiOCl}, \quad$ (b), (e) $12.5 \%$ F substitution: $\mathrm{TiO}_{7 / 8} \mathrm{~F}_{1 / 8} \mathrm{Cl}$, and (c), (f) $12.5 \% \mathrm{~S}$ substitution: $\mathrm{TiOCl}_{7 / 8} \mathrm{~S}_{1 / 8}$. In (a), (b), and (c) the total Ti-resolved DOS are shown. The grey (orange) curve denotes the DOS for the Ti ion nearest to the doping ion (Ti2 for the Na- and F-doped cases, Ti3 for the S-doped case). The black (blue) curve denotes the DOS for the rest of Ti ions. In (d), (e), and (f) the corresponding orbital-resolved DOS are shown. Below $E_{f}$ the contributions of Ti2 for the electron-doped cases are explicitly marked.

The remaining uncertainty is given by the various combinations of locating $\mathrm{Na}$ atoms into the 8 cages of the supercell. We performed $a b$ initio molecular dynamics for all the different combinations at various doping concentrations and compared the total energies. Fig. 1 presents the final stable lattice structures with lowest total energies for the six $\mathrm{Na}$ concentration values $x$ studied [26]. The supercell structures as a function of $\mathrm{Na}$ doping are shown in the figure and call for further experimental Xray diffraction studies.

In Fig. 2 we present the total density of states (DOS) for the six Na-doped $\mathrm{TiOCl}$ cases considered. The DOS of the undoped case (Fig. 2 (a)) is correctly reproduced with an energy gap around $2 \mathrm{eV}$, and the peak close to the Fermi level is dominantly of $d_{x^{2}-y^{2}}$ character. Here we chose the local coordinate frame as $x\|b, y\| c$ and $z \| a$ with $d_{x^{2}-y^{2}}, d_{x z}, d_{y z}$ forming the $t_{2 g}$ bands and $d_{x y}$, $d_{z^{2}}$ the $e_{g}$ bands. With Na doping (Figs. 2 (b)-(f)) the whole spectral weight is suddenly shifted towards lower energies, and an additional peak with weight $2 x$ appears close to the Fermi level as compared to the undoped case. The separation of the two Ti peaks is about $1 \mathrm{eV}$ for $x=1 / 8$ and a gap always persists at the Fermi level in all Na-doped cases. All the findings are consistent with the experimental observations [16].

In order to understand the microscopic origin of this doped insulating state, we show in Figs. 3 (a) and (d) the orbital-resolved DOS for a Na doping of $x=1 / 8$. Close to the Fermi level, both $d_{x^{2}-y^{2}}$ and $d_{x z}$ orbitals contribute to the DOS. This can be understood by performing a rough energy estimation in the atomic limit when one additional electron is added to the Ti $\mathrm{t}_{2 g}$ bands. While the change of total energy is $U+2 J_{\mathrm{H}}$ if the additional electron occupies the $d_{x^{2}-y^{2}}$ orbital, it is $U-J_{\mathrm{H}}+\Delta$ if the 
electron occupies the $d_{x z}$ orbital where $\Delta$ is the orbital excitation from $d_{x^{2}-y^{2}}$ to $d_{x z}$, and $J_{\mathrm{H}}$ is the Hund's rule coupling. Since $\Delta \sim 0.1 \mathrm{eV}$ and $J_{\mathrm{H}} \sim 1 \mathrm{eV}$, adding the additional electron to the $d_{x z}$ orbital is always preferable. This analysis indicates the importance of the Hund's rule coupling in the doping case.

The presented DFT results point to a multi-band scenario. With the help of $N$-th order muffin tin orbital (NMTO) downfolding 27] we found that the hopping integrals within $d_{x z}$ orbitals along $a$ and within one $\mathrm{TiO}$ bilayer are comparable to the hopping integrals within $d_{x^{2}-y^{2}}$ orbitals along $b$, indicating that the interaction network is two-dimensional under doping. Since the exchange interactions among local spins along $a$ and within one TiO bilayer are weakly ferromagnetic [13], the doped electron would in principle hop freely in the system 28] and would result in a metallic state. This is though in contradiction with the insulating state obtained both in our DFT calculations and in experimental observations.

Therefore, to further elucidate the possible mechanism for the insulating state upon doping, we compare the crystal field splitting of the undoped case with that of the 1/8-Na-doped case as shown in Figs.4 (a) and (b). In the undoped case all $8 \mathrm{Ti}$ ions in the supercell are equivalent and the orbital excitations are the same. Here, the $\mathrm{Ti}$ ions are labelled from Ti1 to Ti8 as shown in Fig. 1] (b). In the 1/8-Na-doped case, all Ti ions become inequivalent and the crystal field splittings are different from site to site. Most importantly, the splittings of $t_{2 g}$ orbitals on the Ti2 (closest Ti to Na) become strikingly small, which makes it possible to trap the additional electron into $d_{x z}$ orbitals on this Ti site. This finding is consistent with the partial DOS shown in Figs. 3 (a) and (d) where the peaks near the Fermi level are mainly contributions from the $d_{x^{2}-y^{2}}$ and $d_{x z}$ orbitals of Ti2. The peak at lower energies is from the rest of $\mathrm{Ti}$ ions (all denoted by $\mathrm{Ti} \overline{2}$ ) and is of purely $d_{x^{2}-y^{2}}$ character. Further investigations of the crystal field splittings and partial DOS for the other $\mathrm{Na}$ doping concentrations reveal that the same mechanism can be applied to the observed insulating states, i.e., each $\mathrm{Na}$ ion strongly modifies the lattice structure locally, reduces the crystal field splitting of the $t_{2 g}$ orbitals on the closest $\mathrm{Ti}$ ion and leads to localization of the doped electron, which reduces the $\mathrm{Ti}^{3+}\left(3 d^{1}\right)$ to $\mathrm{Ti}^{2+}$ $\left(3 d^{2}\right)$ and prevents conduction. Furthermore, long-range Coulomb repulsion becomes effective due to the appearance of mixed-valence state of $\mathrm{Ti}^{3+}$ and $\mathrm{Ti}^{2+}$ and further stabilizes the symmetry-breaking insulator. Within the point-charge approximation [16], the combination of crystal field splitting and the long-range Coulomb interaction can account for the gap of $1 \mathrm{eV}$.

We have also considered the effect of substituting $\mathrm{O}$ by F (Fig. 3 (b), (e)), Cl by S (Fig. 3 (c), (f)), and Ti by $\mathrm{V}$ or Sc (not shown) in the undoped TiOCl at concentrations of $1 / 8$, leading to $\mathrm{TiO}_{7 / 8} \mathrm{~F}_{1 / 8} \mathrm{Cl}, \mathrm{TiOCl}_{7 / 8} \mathrm{~S}_{1 / 8}$, $\mathrm{Ti}_{7 / 8} \mathrm{~V}_{1 / 8} \mathrm{OCl}$ and $\mathrm{Ti}_{7 / 8} \mathrm{Sc}_{1 / 8} \mathrm{OCl}$. The atomic positions

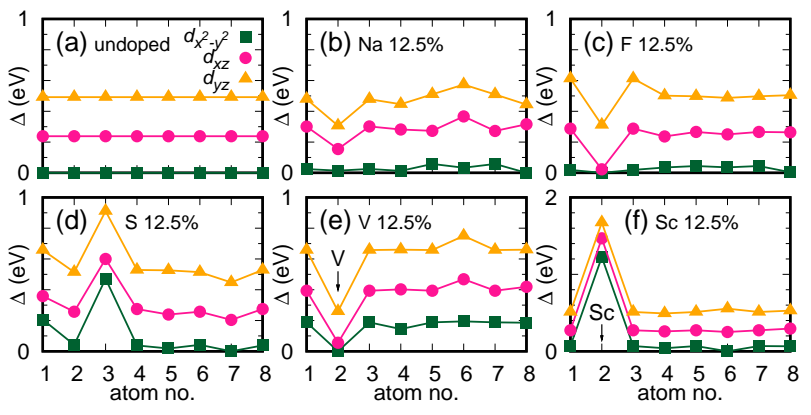

FIG. 4: (Color online) $\mathrm{t}_{2 g}$ crystal field splitting energies obtained from GGA calculations. The horizontal axis denotes $8 \mathrm{Ti}$ ions in the supercell labelled by numbers from 1 to 8 as shown in Fig. 1 (b). (a) $\mathrm{TiOCl}$, (b) $\mathrm{Na}_{1 / 8} \mathrm{TiOCl}$, (c) $\mathrm{TiO}_{7 / 8} \mathrm{~F}_{1 / 8} \mathrm{Cl}$, (d) $\mathrm{TiOCl}_{7 / 8} \mathrm{~S}_{1 / 8}$, (e) $\mathrm{Ti}_{7 / 8} \mathrm{~V}_{1 / 8} \mathrm{OCl}$, and (f) $\mathrm{Ti}_{7 / 8} \mathrm{Sc}_{1 / 8} \mathrm{OCl}$.

where the substitutions are made (without loss of generality) are specified in Fig. 1] (a). In all the doped cases gaps still open at the Fermi level. The DOS of the Fdoped case (Fig. 3 (b), (e)) is similar to that of the Nadoped case with a double peak structure below the Fermi level, since both are electron doping processes. The similarities can be also observed in the crystal field splittings (Fig. $4(\mathrm{c})$ ). However, in the F-doped case the Ti2 $d_{x^{2}-y^{2}}$ and $d_{x z}$ orbitals are almost degenerate.

Substituting $\mathrm{Cl}$ by $\mathrm{S}$ implies taking out an electron from the $d_{x^{2}-y^{2}}$ orbital (hole doping), and one would expect the system to be metallic. However, from Fig. 4 (d), we find that the on-site orbital energies of Ti3 (Fig. 4(d)) which is closest to the substituted $\mathrm{S}$ ion are significantly raised due to the large distortion of the lattice structure, again leading to a localized state for the doped hole. In this case, no spectral weight from the Ti3 $3 d$ orbitals is detected close to the Fermi level (Fig. 3 (c)). Interestingly, the Ti1 $d_{x^{2}-y^{2}}$ orbital energy is almost degenerate with the Ti7 $d_{x z}$ orbital energy, indicating the importance of on-site Coulomb interaction which avoids double occupation on each Ti ion. This leads to a DOS below the Fermi level of only $d_{x^{2}-y^{2}}$ character (Fig. 3 (f)). On the other hand, doping with $\mathrm{S}$ might result in the formation of $\mathrm{S}_{2}^{2-}$ ions, which are isoelectronic to two $\mathrm{Cl}^{-}$ions and do not contribute to doping. The system will then remain insulating. Finally, substituting Ti by V (electron doping) and Sc (hole doping) we observe a similar effect as seen in the previous electron- and hole-doped cases. The onsite orbital energies are much lower (higher) on the $\mathrm{V}$ (Sc) ion than on the Ti ion (Figs. 4(e) and (f)), which can again account for the localization of the doped electron (hole) and the absence of a metallic state. However, the oxidations are all $+3\left(\mathrm{Ti}^{3+}, \mathrm{V}^{3+}, \mathrm{Sc}^{3+}\right)$, indicating that the role of long-range Coulomb interaction is negligible.

From the above analysis, we conclude that the appropriate microscopic model that accounts for the persistence of the insulating state upon doping is given by a 
multiband ionic Hubbard model:

$$
\begin{aligned}
H & =\sum_{i, j, \sigma, \tau \tau^{\prime}} t_{i j, \tau \tau^{\prime}} c_{i \tau \sigma}^{\dagger} c_{j \tau^{\prime} \sigma}+\left(U+2 J_{\mathrm{H}}\right) \sum_{i, \tau} n_{i \tau \uparrow} n_{i \tau \downarrow} \\
& +U \sum_{i, \tau>\tau^{\prime}, \sigma} n_{i \tau \sigma} n_{i \tau^{\prime} \bar{\sigma}}+\left(U-J_{\mathrm{H}}\right) \sum_{i, \tau>\tau^{\prime}, \sigma} n_{i \tau \sigma} n_{i \tau^{\prime} \sigma} \\
& +\sum_{i, \sigma, \tau} \Delta_{i, \tau} n_{i \tau \sigma}+\sum_{i, j} V_{i j} n_{i} n_{j} .
\end{aligned}
$$

The first term describes the hopping between Ti sites $i, j$ within the three $\mathrm{t}_{2 g}$ orbitals $\left(\tau, \tau^{\prime}\right)$, the second to fourth terms are the intra- and inter-orbital Coulomb interactions and the Hund's rule coupling, respectively, the fifth term the crucial on-site orbital energies $\Delta_{i, \tau}$, and the last term the effective long-range intersite Coulomb interaction $V_{i j}$. Upon Na doping, $\Delta_{i, \tau}$ differs from site to site which forms a local trapping potential and leads to a coexistence of $\mathrm{Ti}^{3+}$ and $\mathrm{Ti}^{2+}$ stabilized by $V_{i j}$. Then, a doping-induced phase transition from undoped Mott insulator to doped insulator with charge disproportionation occurs. While a similar transition has been extensively investigated in the one-band case [17], inclusion of orbital degrees of freedom may result in an even richer phase diagram [29, 30].

Although our DFT calculations are only performed for several commensurate $\mathrm{Na}$ doping cases, we argue that the scenario for this insulating state under doping can be applied to all doping concentrations: the Na ion enters an individual cage consisting of $5 \mathrm{Cl}$ and $1 \mathrm{O}$ ions and creates a trapping potential reducing the crystal field splitting of the $t_{2 g}$ orbitals on the closest $\mathrm{Ti}$ ion and resulting in a localized state for the doped electron with the help of long-range Coulomb interaction.

Finally, with the understanding of the mechanism for the insulating state, we propose two ways to metallize TiOCl by doping which would favor a possible superconducting state in a doped Mott insulator by suppressing other symmetry-breaking states. The first idea is to avoid the strong modification of crystal field splitting and the formation of a trapping potential due to the lattice deformation induced by the cation or anion. Therefore, a possible direction is to intercalate $\mathrm{Na}$ together with organic ligands to prevent the doped $\mathrm{Na}$ from entering the cage, in the spirit of intercalating organosolvated Li into $\beta$-HfNCl [6]. A second practicable way is to apply external pressure [14, 15] after carrying out electron doping. Due to the appearance of the additional peak and the reduction of the gap under doping, the doped system becomes much easier to metallize under pressure than the undoped one.

In conclusion, we performed DFT calculations on doped $\mathrm{TiOCl}$ and unveiled the microscopic origin of the observed insulating state upon doping. The superlattice structures at different $\mathrm{Na}$ doping concentrations and the effects of different substitutions are predicted. We find that the opportunity to metallize $\mathrm{TiOCl}$ or even to make it superconducting still remains. Moverover, the insulator-to-insulator transition itself has been extensively discussed by tuning the correlation strength [17]. Here, we open a new way to induce the insulator-insulator transition by doping. Finally, our proposed model may contain novel phases which calls for further investigations by many-body techniques [29, 30$]$.

We thank R. Claessen, M. Sing, M. Knupfer, M. AbdElmeguid, and C. Gros for useful discussions. We thank the Deutsche Forschungsgemeinschaft for financial support through the TRR/SFB 49, FOR 412 and Emmy Noether programs, and we acknowledge support by the Frankfurt Center for Scientific Computing.

[1] H. Eisaki, et al., Phys. Rev. B 69, 064512 (2004).

[2] Y. Tokura, et al., Nature 337, 345 (1989).

[3] Y. Kamihara, et al., J. Am. Chem. Soc. 130, 3296 (2008).

[4] M. Rotter, et al., Phys. Rev. Lett. 101, 107006 (2008).

[5] Y. Mizuguchi, et al., Appl. Phys. Lett. 93, 152505 (2008).

[6] S. Yamanaka, et al., Nature 392, 580 (1998).

[7] K. Takada, et al., Nature 422, 53 (2003).

[8] A. Seidel et al., Phys. Rev. B 67, 020405(R) (2003); T. Saha-Dasgupta et al., Phys. Rev. B 71, 153108 (2005).

[9] M. Shaz et al., Phys. Rev. B 71, 100405(R) (2005);

[10] R. Rückamp et al., Phys. Rev. Lett. 95, 097203 (2005).

[11] M. Hoinkis, et al., Phys. Rev. B 72, 125127 (2005).

[12] E. T. Abel et al., Phys. Rev. B 76, 214304 (2007).

[13] Y. Z. Zhang, et al., Phys. Rev. B. 78, 205104 (2008).

[14] C. A. Kuntscher et al., Phys. Rev B 78, 035106 (2008).

[15] Y. Z. Zhang, et al., Phys. Rev. Lett. 101, 136406 (2008).

[16] M. Sing et al., arXiv:0905.1381.

[17] M. Fabrizio, et al., Phys. Rev. Lett. 83, 2014 (1999); A. Garg et al., Phys. Rev. Lett., 97, 046403 (2006); S. Kancharla, E. Dagotto, Phys. Rev. Lett., 98, 016402 (2007).

[18] R. Car, M. Parrinello, Phys. Rev. Lett. 55, 2471 (1985).

[19] P. E. Blöchl, Phys. Rev. B 50, 17953 (1994).

[20] M. Parrinello, A. Rahman, Phys. Rev. Lett. 45, 1196 (1980).

[21] L. Pisani, R. Valentí, Phys. Rev. B 71, 180409(R) (2005).

[22] $U=4.5 \mathrm{eV}$ and $J_{\mathrm{H}}=1.0 \mathrm{eV}$ were chosen within GGA $+U$ to reproduce the correct gap of $2 \mathrm{eV}$ for undoped TiOCl.

[23] It is well-known that GGA + U can describe the Mott insulator by imposing suitable magnetic/orbital ordering and other insulators with symmetry breaking. Its applicability to our study is justified since TiOCl retains insulating behaviour upon doping. For a review of methods, see K. Held, Adv. Phys. 56, 829 (2007).

[24] Y. Z. Zhang, et al., J. Phys. Soc. Jpn. 74, 2153 (2005).

[25] L. Pauling, J. Am. Chem. Soc. 51, 1010 (1929).

[26] Other metastable configurations may coexist with the groundstate one at finite temperature, which may account for the soft gap observed experimentally at higher Na-doping. Here, we only aim at the ground state.

[27] T. Saha-Dasgupta, O. K. Andersen, Phys. Rev. B 62, R16219 (2000).

[28] C. Zener, Phys. Rev. 82, 403 (1951).

[29] L. de'Medici, et al., Phys. Rev. Lett. 102, 126401 (2009).

[30] H. Lee, et al., Phys. Rev. Lett. 104, 026402 (2010). 